\title{
Preventing the suspension of dental clinics by minimizing the risk of SARS-CoV-2 transmission during dental treatment
}

\author{
Sylwia Czajkowska ${ }^{1, A, B, D}$, Natalia Potempa ${ }^{1, D, E}$, Joanna Rupa-Matysek2, ${ }^{2, E, F}$, Anna Surdacka ${ }^{1, E, F}$ \\ ${ }^{1}$ Department of Conservative Dentistry and Endodontics, Poznan University of Medical Sciences, Poland \\ ${ }^{2}$ Department of Hematology and Bone Marrow Transplantation, Poznan University of Medical Sciences, Poland \\ A - research concept and design; $\mathrm{B}$ - collection and/or assembly of data; $\mathrm{C}$ - data analysis and interpretation; \\ $D$ - writing the article; $E$ - critical revision of the article; $F$ - final approval of the article
}

Address for correspondence

Natalia Potempa

E-mail: potempa.natalia@gmail.com

Funding sources

None declared

Conflict of interest

None declared

Received on December 10, 2020

Reviewed on February 11, 2021

Accepted on February 18, 2021

Published online on September 30, 2021

Cite as

Czajkowska S, Potempa N, Rupa-Matysek J, Surdacka A.

Preventing the suspension of dental clinics by minimizing the risk

of SARS-CoV-2 transmission during dental treatment. Dent Med

Probl. 2021;58(3):397-403. doi:10.17219/dmp/133442

DOI

$10.17219 / \mathrm{dmp} / 133442$

Copyright

○) 2021 by Wroclaw Medical University

This is an article distributed under the terms of the

Creative Commons Attribution 3.0 Unported License (CC BY 3.0)

(https://creativecommons.org/licenses/by/3.0/).

\begin{abstract}
Due to a high risk of the transmission of severe acute respiratory syndrome coronavirus 2 (SARS-COV-2) during dental work, the coronavirus disease 2019 (COVID-19) pandemic has had a considerable influence on the functioning of dental clinics. The elevated risk of transmission is related to the production of aerosol containing secretions from the upper respiratory tract that is produced during dental procedures.

The purpose of this narrative review was to present the current knowledge concerning COVID-19 and to propose methods for reducing the spread of the virus. Dental staff should follow the current guidelines and ensure safety at work through the use of personal protective equipment (PPE), including FFP2/FFP3 filter masks, the implementation of the screening protocols and telephone consultations as well as the appropriate preparation of dental practices and patient waiting areas. In addition, it is essential to reduce the number of people simultaneously occupying one building by effectively planning visiting times or discouraging patients from accompanied visits. Procedures that may contribute to production of the aerosol should also be limited.
\end{abstract}

Keywords: aerosols, COVID-19, dental staff, dentists, personal protective equipment 


\section{Introduction}

The coronavirus disease 2019 (COVID-19) pandemic was officially declared by the World Health Organization (WHO) on March 11, 2020. ${ }^{1}$ No previous epidemics, including those caused by coronaviruses such as severe acute respiratory syndrome coronavirus 1 (SARS-CoV-1) or Middle East respiratory syndrome coronavirus (MERS$\mathrm{CoV})$, have had such a wide-ranging impact on the economy, and on the functioning of dental clinics and other healthcare facilities.

The SARS-CoV-2 virus has been the subject of many studies and, to this day, we do not know the long-term effects of the infection, which appear to be very diverse. ${ }^{2}$ According to WHO reports, the most common symptoms of infection are increased body temperature, fatigue and a dry cough. Less often, there are instances of loss of taste and/or smell, nasal congestion, conjunctivitis manifested by the redness of the eyes, muscle and joint pain, sore throat, headache, skin rash, diarrhea, nausea and vomiting, chills, and dizziness. Patients with a severe COVID-19 infection may also experience confusion, the loss of appetite, or persistent chest pain or tightness. ${ }^{3}$ Studies have shown that the severity of the disease and the risk of mortality are influenced by the patient's age (over 65 years), the presence of pre-existing diseases and obesity. ${ }^{2,4-8}$ Coronavirus disease 2019 can also affect the whole body, including the parameters of the coagulation system. For example, studies have outlined the possible appearance of disseminated intravascular coagulation (DIC), even in patients with a congenital hemorrhagic diathesis. ${ }^{10}$ Laboratory studies of patients with SARS-CoV-2 infection have shown an increase in prothrombin time, a decrease in platelet counts, an elevation in fibrinogen levels, a rise in D-dimer levels, and increases in both coagulation factor VIII and von Willebrand factor. ${ }^{8,11-14}$

A special role in the transmission of SARS-CoV-2 is played by the aerosol produced during dental procedures, containing secretions from the upper respiratory tract. The virus itself can be transmitted by both symptomatic and asymptomatic individuals. ${ }^{15}$ Due to the character of dental work, dental clinics can be a place of a high risk of the transmission of the virus. The following review is narrative in nature, and aims to present information on viral transmission in dental clinics and to summarize the guidelines for dental treatment during the COVID-19 pandemic.

\section{Material and methods}

The criteria for the inclusion of studies and articles in the present review are listed below. The review has a narrative structure; it was conducted by 4 researchers and is limited to studies related to oral hygiene, dental care, the SARS-CoV-2 virus, and the functioning of dental clin- ics in the era of the SARS-CoV-2 pandemic. The studies included in the analysis concerned guidelines, recommendations, case reports, and academic work, regardless of the original language, year and status of the publication, as long as there was sufficient evidence provided and the studies were related to the subject. The PubMed/MEDLINE database was used to obtain information from the National Library of Medicine (NLM) based on 2 search strategies. One strategy used Medical Subject Headings $(\mathrm{MeSH})$, whereas the other one referred to the keywords in the text. The search involved items published up to November 21, 2020. When a more directed search was needed, related articles were evaluated. Recommendations from international organizations, such as WHO, the Polish Dental Society (PTS), the American Dental Association (ADA), the Royal College of Surgeons of England, the World Federation of Hemophilia (WFH), and the Centers for Disease Control and Prevention (CDC), were also examined. In addition, the bibliographic references in all of the searched reviews and studies were screened for additional research reports. Due to the limited number of original articles, and the lack of randomized and quasirandomized studies published in this field prior to the end date of the search process, the results were presented in the form of a narrative review. The aim was to compile studies, guidelines and case reports, and make it easier for dentists to perform procedures and work in the rapidly changing epidemiological situation. Systematic presentation of all data is necessary due to the ongoing $\mathrm{CO}$ VID-19 pandemic and the risk of the transmission of the virus during dental treatment. Eventually, 40 articles and 8 websites were included.

\section{Discussion}

The SARS-CoV-2 pandemic has had a great impact on access to dental care and on dental services. ${ }^{16,17}$ According to a study conducted at the Medical University of Silesia in Katowice, Poland, during the government lockdown in the spring of 2020 , as many as $71.2 \%$ of dentists decided to suspend clinical practice. ${ }^{16}$ The main reasons for the cessation of work were insufficient access to personal protective equipment (PPE) and subjective feelings about COVID-19, including a sense of anxiety and uncertainty. In addition, a decrease in the number of patients in dental clinics was observed as compared to the period before the pandemic was announced. ${ }^{16}$ Reducing the number of follow-up visits and the postponements of the scheduled treatment can have a sizable impact on the oral health of the population, thus contributing to an increase in the number of patients with acute periapical tissue inflammation, abscesses or acute pulp inflammation. ${ }^{17,18}$

Scientific research confirms that there is a high risk of virus transmission in dental clinics. ${ }^{7,16,19}$ This is related to the performance of numerous procedures leading to 
the formation of aerosol that contains microorganisms, drops of saliva, blood, water, and other contaminants. ${ }^{20}$ In addition, close contact between the dentist and the patient promotes the transmission of potential infection and causes dental staff to be a source of cross-transmission. ${ }^{21}$ National and international organizations, such as ADA, the Royal College of Surgeons of England, CDC, and PTS, are monitoring and evaluating the spread of the virus on an ongoing basis as well as constantly providing recommendations and guidelines on the functioning of dental practices. According to the current epidemiological situation, it is important to monitor and adapt dental clinics to the latest guidelines.

Many international associations state that the primary way to reduce the risk of the transmission of the virus is through the use of PPE. Surgical masks do not provide full protection against the inhalation of infectious agents, but constitute a barrier between mucous membranes and the sprayed drops. ${ }^{20}$ The best respiratory protection would be the use of masks with a filter. These are marked with symbols N95, N99 and N100 according to American standards, or FFP1, FFP2 and FFP3 according to European classifications. The quality of a filter marked with the N95 standard is assumed to be nearly equal to that of a filter marked with the European symbol FFP2. ${ }^{21}$ Based on the analysis of scientific studies and recommendations, authors encourage the use of masks of at least N95 or FFP2 standard. In the case of a mask with an exhaust valve, an additional mask is needed to cover the valve. ${ }^{20}$ Researchers also point out that fabric face guards do not constitute individual protection. ${ }^{20}$ Additionally, glasses/ face shields and medical clothing are also advised as part of PPE, and it is suggested to use a long-sleeved cover under the gloves to protect the forearms. ${ }^{21}$

It should be noted that SARS-CoV-2 can persist on surfaces such as stainless steel or plastic and can remain infectious for up to 3 days at room temperature. ${ }^{22}$ Therefore, frequent surface and air disinfection is of vital importance in preventing infection. Items in the waiting room that are not suitable for regular cleaning and sterilization, such as magazines or toys, should be removed from the room. ${ }^{20,23}$ For coronaviruses, it is preferable to use a product containing $62-71 \%$ ethanol, $0.1 \%$ sodium hypochlorite or $0.5 \%$ hydrogen peroxide for $1 \mathrm{~min} .{ }^{24} \mathrm{Hand}$ hygiene should be carried out with soap and water, followed by the application of a product containing 70-90\% alcohol. ${ }^{21}$ Hand sanitizers, together with instructions for use, should also be provided to patients before entering a medical room. ${ }^{20}$ Air disinfection also plays an essential role in the fight against the pandemic. An interesting solution seems to be the use of ozone equipment in dental offices between visits or in the waiting room after work. Plasma devices may also be used, as they are characterized by a high level of disinfection capability. ${ }^{21}$ Modern technologies can also be helpful in reducing the number of bacteria, viruses and fungi on flat surfaces. High effica- cy has been demonstrated with the use of hydrogen peroxide fumigation. ${ }^{21}$ Depending on the financial capacity of the clinic, dentists should consider the daily use of disinfection devices. It is crucial to follow the recommendations of the device manufacturers, and it should be noted that some methods of disinfection can affect the quality and durability of various items in dental clinics. In addition, the specific methods may vary, for instance, in the time of room ventilation or the length of the disinfection process. When ultraviolet (UV) lamps are applied, surface disinfection occurs $8 \mathrm{~h}$ after switching the device on and it is recommended to leave the room during this time. ${ }^{25}$ A shorter operating time is needed for ozone generators; depending on the room size, ozone disinfection takes an average of 4-5 h. However, it is essential not to apply other disinfection equipment, including UV lamps, at the same time. ${ }^{25}$ The main disadvantages of ozone generators are the possibility of causing damage and discoloration to plastic or rubber components as well as the inadequate selection of the ozone dose for the room size. ${ }^{25}$ When ozone disinfection is completed, it is recommended to ventilate dental offices and avoid entering the rooms for up to $2 \mathrm{~h}$ after the process. ${ }^{25}$ Significantly shorter ventilation times (30 min on average) and disinfection times (about $10 \mathrm{~min}$ ) are possible with fumigators. ${ }^{25}$

Until the end of the acute phase of the pandemic, recommendations also include the admission of only urgent patients to the dentist's office. ${ }^{26}$ In any case, it is necessary to assess the risk associated with the postponement of treatment and the risk associated with the possible transmission of the virus. ${ }^{20}$ It is suggested to consider providing telephone consultations, and the possibility of preparing electronic consents and prescriptions. Additionally, telephone consultations should be recorded in the patient's medical records. ${ }^{27}$ According to a study published in April 2020 by WHO, concerning the use of analgesics, there is no evidence to indicate any influence of non-steroidal anti-inflammatory drugs (NSAIDs) on the occurrence of serious adverse events, long-term survival or quality of life of patients with confirmed COVID-19. ${ }^{28}$ The previous WHO statement, prior to this study, discouraged the use of NSAIDs in this group of patients. However, it should be noted that there are particular diseases where these drugs are contraindicated. It refers to patients with congenital hemorrhagic diathesis, as NSAIDs affect platelet aggregation. ${ }^{29}$ In such conditions, the approved analgesic is paracetamol (acetaminophen) at a dose not exceeding $60 \mathrm{mg} / \mathrm{kg} /$ day or $3 \mathrm{mg} /$ day. The American Dental Association issued recommendations to help determine what constitutes an emergency case. ${ }^{30}$ This classification system, based on the time required to intervene, divides emergency situations into 3 categories (Table 1). Other kinds of dental treatment that are not listed in the table, such as preventive or hygienic procedures, are regarded routine treatment that can be delayed. Numerous publications recommend that the appointment at the doctor's 
Table 1. Modified dental emergency classification by the American Dental Association (ADA) $)^{31}$

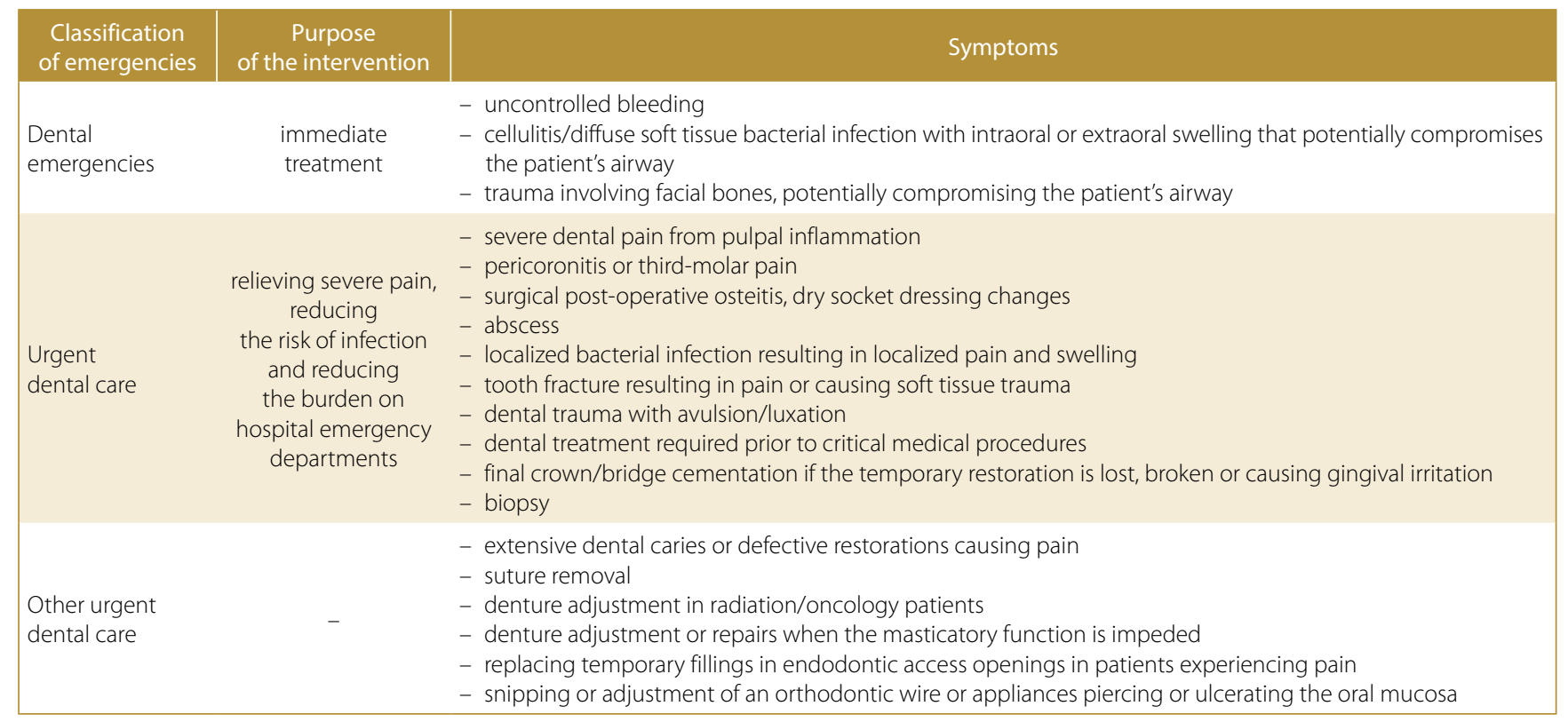

office should be made by telephone in order to verify the reasons for a visit and to apply a screening protocol. ${ }^{20,23}$ The authors of this article support this opinion and state that the selection of patients by phone may also enable the gathering of detailed information on dental problems and oral health. On the basis of personal experience and the recommendations of international societies, it is suggested to obtain any information regarding the patient's close contacts with any persons tested positive for COVID-19 or being under quarantine. Moreover, it is advisable to collect information regarding the presence of symptoms suggestive of respiratory infections (cough, elevated body temperature, shortness of breath) or travel abroad to high-risk areas. ${ }^{7,15,19,21,27,31}$ Of note, the confirmation of a negative test result for SARS-CoV-2 does not relieve medical personnel of extreme caution and PPE should be applied in all cases. Patients with confirmed COVID-19 should be referred to a treatment center designated for receiving patients from this group ${ }^{17,31}$ or, if possible, it is recommended to postpone dental treatment until isolation or quarantine is completed. ${ }^{20}$ Furthermore, it is important to adequately space appointments to allow time for full cleaning and disinfection after dental surgery, ${ }^{21}$ and to minimize the number of patients in the waiting room. ${ }^{20,23}$ In addition, it is suggested to place the seats in the lobby in such a way as to maintain the recommended social distance between individuals. ${ }^{20}$ This will not only decrease the risk of viral transmission, but may also have a positive impact on the patients' sense of safety, as it has been shown that the highest risk of the transmission of COVID-19 infection is associated with close contact with another patient. ${ }^{32}$

In order to minimize the risk of the transmission of the virus in the dental clinic, the patient should be asked to attend alone or only with a person necessary to carry out the visit, ${ }^{20,23}$ such as a legal guardian in the case of a child or an incapacitated person. The patient should be informed that, when entering a medical institution and during screening, both he/she and any accompanying person are obliged to wear a mask that covers the mouth and the nose. ${ }^{20,23}$ It is the responsibility of the healthcare professional to ensure that each entering person complies with the recommendations and to conduct a test for symptoms (including the measurement of body temperature) consistent with SARS-CoV-2 infection. ${ }^{20,23}$ It is important to document the lack of symptoms and, in the case of elevated body temperature with the absence of other general symptoms associated with COVID-19, to draw attention to any possible association with a dental diagnosis. ${ }^{20}$

In the dental clinic, before examination, the patient should be instructed to rinse their mouth for a minimum of $30 \mathrm{~s}$ with a disinfectant-based preparation. The proposed solutions are $0.2 \%$ chlorhexidine, $2 \%$ Listerine $^{\circledR}$, $2 \%$ iodine povidone, or $0.5-1 \%$ hydrogen peroxide.,21,31 In addition, to eliminate viruses, it is advised to consider the use of modern technologies, such as the ozone therapy or the laser therapy. The neodymium-doped yttrium aluminum garnet (Nd:YAG) laser $(1,064 \mathrm{~nm})$ has been shown to have an antibacterial effect that is dependent on the thermal warming of the bacterial environment and local heating inside pathogens. ${ }^{33}$ These properties are used in dentistry primarily in the sterilization and disinfection of root canals. In addition, the Nd:YAG laser is the most recommended laser in endodontics. Studies show that the application of the Nd:YAG laser reduces the number of Enterococcus faecalis and Escheria coli bacteria by $99.92 \% .{ }^{33}$ During this particular laser therapy, it is important to use a fiberglass tip with a diameter of approx. $200 \mu \mathrm{m}$, placed about 1-2 $\mathrm{mm}$ from the apex. The tip should be moved toward the crown with circu- 
lar and gradual movements. The laser is used 4 times for 5-10 s with 20-second breaks. Safe parameters for the Nd:YAG laser are $100 \mathrm{~mJ}, 15 \mathrm{~Hz}$ and $1.5 \mathrm{~W}^{33} \mathrm{An}$ other method for tissue disinfection in the mouth is the ozone therapy in the form of ozonated water or oil. ${ }^{34}$ The use of ozone water reduces the number of Streptococcus mutans bacteria in the plaque, and thus contributes to the prevention of the progression of caries. ${ }^{34}$ Moreover, ozone can also be used as an additional therapy to disinfect root canals and treat gingivitis, periodontitis, or even osteonecrosis. ${ }^{34}$

The SARS-CoV-2 virus has been identified in the airway, ${ }^{35}$ saliva ${ }^{36}$ and gastrointestinal tract. ${ }^{36,37}$ For this reason, it is not recommended to take intraoral X-rays, ${ }^{7,30}$ as it can provoke a gag reflex or increase salivation. If a radiological diagnosis is required, extraoral imaging, such as pantomographic $\mathrm{X}$-ray or cone-beam computed tomography $(\mathrm{CBCT})$, is preferred. It is permissible to take an intraoral photograph, but the dentist should choose bitewing radiography. If the doctor decides to take a dental photograph without a positioner, the patient should disinfect their hands before holding the X-ray film. ${ }^{21}$ If a positioner is used, it must be sterilized after each use. ${ }^{21}$ When taking intraoral photographs, it is suggested to use a double cover on the radiological sensor to reduce the risk of contamination. ${ }^{15}$ Avoiding small-format pictures refers primarily to patients with confirmed SARS-CoV-2 virus infection. ${ }^{38}$ In such cases, intraoral radiograms should be used in patients who cooperate and can breathe well through the nose. In order to control the risk of the transmission of the virus, the patient should be informed of a possible gag reflex and asked about previous problems with taking an intraoral photograph..$^{38}$

Patients who report to dental clinics during a pandemic are primarily patients in pain. Additionally, patients with concomitant diseases deserve special attention. In situations where urgent intervention is necessary, patients with congenital hemorrhagic disorders are particularly difficult cases, as a pre-treatment consultation with a hematologist is often necessary. Extraction is a procedure involving the risk of delayed or prolonged bleeding. ${ }^{39-41}$ In this case, it should be remembered that an alternative is endodontic treatment. ${ }^{42}$ During such a procedure, it is essential to use an apex locator and to be especially aware of work duration times. ${ }^{42}$ This can prevent possible bleeding from periodontal fibers. The proposed dressing used between visits is calcium hydroxide, which has drying properties. ${ }^{42,43}$ In the presence of residual vital pulp in the bleeding canal, the rinsing protocol should be supplemented with sodium hypochlorite solution. ${ }^{39,40}$ Procedures requiring extreme caution and the supplementation of the missing factor are mandibular nerve block anesthesia and infiltration anesthesia from the lingual side. ${ }^{43,44}$ Intrapulpal, interdental or infiltration anesthesia from the buccal side and mental nerve block anesthesia can be considered safe. ${ }^{45}$
The scheduled visits, such as prosthetic and orthodontic treatment, should be postponed until the end of the acute phase of the pandemic. ${ }^{26}$ In order to resist the transmission of the virus, it is recommended to use rubber dams $s^{7,15,23,31}$ and to apply dental suction ${ }^{23}$ whenever possible. Authors recommend paying attention to the type of suction device, as this directly contributes to the amount of aerosol formed in the doctor's office. Obtaining the smallest possible amount of aerosol in the environment requires the use of efficacious suction equipment. ${ }^{46}$ During some procedures, such as the removal of calculus, it is worth considering to replace mechanical tools with hand instruments. ${ }^{23}$ When working with children, the use of atraumatic or minimally invasive procedures should be considered. ${ }^{47}$ An interesting alternative to the traditional cleaning of a dental cavity with rotary tools is a laser. Authors suggest, particularly in the time of the pandemic, that laser application may be of particular importance. While using the Er:YAG laser with the H14 handle (LightWalker; Fotona, Ljubljana, Slovenia), with the parameters set at $300 \mathrm{~mJ}$ energy, $20 \mathrm{~Hz}$ frequency and $6 \mathrm{~W}$ power, and water/air coolant 6/4, less aerosol is produced as compared to the traditional tooth preparation procedures. ${ }^{46}$ In order to minimize the risk of vomiting during impression-taking procedures, and thus to reduce the transmission of SARS-CoV-2, it is suggested to carefully adjust the proper dental tray before taking the impression, ${ }^{19,48}$ to avoid the use of products of excessively fluid consisten$\mathrm{cy}^{21}$ and to ensure the correct position of the patient ${ }^{48}$; for patients with a developed gag reflex, it is advisable to use a local anesthetic. ${ }^{48}$ Special care should be taken if bleeding in the mouth occurs, as the swallowed or inspired blood may cause vomiting or coughing. ${ }^{21}$ Furthermore, the computer-aided design/computer-aided manufacturing (CAD-CAM) technology is extremely useful today, and it can replace traditional impressions in the future. ${ }^{48}$

\section{Conclusions}

The current COVID-19 pandemic has introduced many restrictions at the individual and social level. The problem has become global and has posed new health and dental challenges. Doctors and dentists are not only obliged to pay attention to the general health of the patient, but also to their own safety and protection against the SARS-CoV-2 virus. Prevention and dental education, which help to maintain oral health, should become essentials, as they minimize the need for dental surgeries and reduce the number of emergency procedures. In the case of an urgent dental intervention, dentists must follow all applicable safety rules to protect themselves, the patient and the dental clinic area. It is also suggested to provide treatment during a single dental visit, while taking preventive measures to reduce the number of consecutive visits. Moreover, it is also important to conduct a detailed 
medical and dental interview, and to manage documentation. Efficacious communication and information flow can be very helpful in preparing the right treatment plan. Given the current situation, every action should be carefully planned and thought out. Oftentimes, the dentist will have to assess the risks associated both with the discontinuation of treatment and with the potential infection with the SARS-CoV-2 virus. In this way, together we can limit the spread of the pandemic.

\section{ORCID iDs}

Sylwia Czajkowska (1) https://orcid.org/0000-0003-3967-9586 Natalia Potempa (1) https://orcid.org/0000-0003-1550-2479 Joanna Rupa-Matysek (10) https://orcid.org/0000-0003-1585-6410 Anna Surdacka (1) https://orcid.org/0000-0001-9952-0230

\section{References}

1. World Health Organization. Twitter thread (March 11, 2020): We have therefore made the assessment that COVID 19 can be characterized as a pandemic. https://twitter.com/who/status/1248354446033199108. Accessed July 11,2020

2. Chen T, Wu D, Chen H, et al. Clinical characteristics of 113 deceased patients with coronavirus disease 2019: Retrospective study. BMJ. 2020;368:m1091. doi:10.1136/bmj.m1091

3. World Health Organization. Coronavirus disease (COVID-19). https://www.who.int/news-room/q-a-detail/coronavirus-diseaseCOVID-19. Accessed November 4, 2020

4. Ioannidis JPA, Axfors C, Contopoulos-loannidis DG. Population-level COVID-19 mortality risk for non-elderly individuals overall and for nonelderly individuals without underlying diseases in pandemic epicenters. Environ Res. 2020;188:109890. doi:10.1016/j.envres.2020.109890

5. Zhou F, Yu T, Du R, et al. Clinical course and risk factors for mortality of adult inpatients with COVID-19 in Wuhan, China: A retrospective cohort study. Lancet. 2020;395(10229):1054-1062. doi:10.1016/S0140-6736(20)30566-3

6. Guan WJ, Liang WH, Zhao Y, et al. Comorbidity and its impact on 1590 patients with COVID-19 in China: A nationwide analysis. Eur Respir J. 2020;55(5):2000547. doi:10.1183/13993003.00547-2020

7. Jamal $\mathrm{M}$, Shah $\mathrm{M}$, Almarzooqi $\mathrm{SH}$, et al. Overview of transnational recommendations for COVID-19 transmission control in dental care settings. Oral Dis. 2020. doi:10.1111/odi.13431

8. Wang D, Hu B, Hu C, et al. Clinical characteristics of 138 hospitalized patients with 2019 novel coronavirus-infected pneumonia in Wuhan, China. JAMA. 2020;323(11):1061-1069. doi:10.1001/jama.2020.1585

9. Liang W, Guan W, Chen R, et al. Cancer patients in SARS-CoV-2 infection: A nationwide analysis in China. Lancet Oncol. 2020;21(3):335-337. doi:10.1016/S1470-2045(20)30096-6

10. Dorgalaleh A, Dabbagh A, Tabibian S, et al. Patients with congenital bleeding disorders appear to be less severely affected by SARS-CoV-2: Is inherited hypocoagulability overcoming acquired hypercoagulability of coronavirus disease 2019 (COVID-19)? Semin Thromb Hemost. 2020;46(7):853-855. doi:10.1055/s-0040-1713435

11. Wu L, O'Kane AM, Peng H, Bi Y, Motriuk-Smith D, Ren J. SARS-CoV-2 and cardiovascular complications: From molecular mechanisms to pharmaceutical management. Biochem Pharmacol. 2020;178:114114. doi:10.1016/j.bcp.2020.114114

12. Lillicrap D. Disseminated intravascular coagulation in patients with 2019-nCoV pneumonia. J Thromb Haemost. 2020;18(4):786-787. doi:10.1111/jth.14781

13. Adam EH, Zacharowski K, Miesbach W. A comprehensive assessment of the coagulation profile in critically ill COVID-19 patients. Thromb Res. 2020;194:42-44. doi:10.1016/j.thromres.2020.06.026

14. Varga Z, Flammer AJ, Steiger $P$, et al. Endothelial cell infection and endotheliitis in COVID-19. Lancet. 2020;395(10234):1417-1418. doi:10.1016/S0140-6736(20)30937-5

15. Baghizadeh Fini M. What dentists need to know about COVID-19. OralOncol.2020;105:104741.doi:10.1016/j.oraloncology.2020.104741
16. Tysiąc-Miśta $M$, Dziedzic $A$. The attitudes and professional approaches of dental practitioners during the COVID-19 outbreak in Poland: A cross-sectional survey. Int J Environ Res Public Health. 2020;17(13):4703. doi:10.3390/ijerph17134703

17. Guo H, Zhou Y, Liu X, Tan J. The impact of the COVID-19 epidemic on the utilization of emergency dental services. J Dent Sci. 2020;15(4):564-567. doi:10.1016/j.jds.2020.02.002

18. Petrescu NB, Aghiorghiesei O, Mesaros A, et al. Impact of COVID-19 on dental emergency services in Cluj-Napoca metropolitan area: A cross-sectional Study. Int J Environ Res Public Health. 2020;17(21):7716. doi:10.3390/ijerph17217716

19. Ge ZY, Yang LM, Xia JJ, Fu XH, Zhang YZ. Possible aerosol transmission of COVID-19 and special precautions in dentistry. J Zhejiang Univ Sci B. 2020;21(5):361-368. doi:10.1631/jzus.B2010010

20. Centers for Disease Control and Prevention. Interim infection prevention and control guidance for dental settings during the coronavirus disease (COVID-19) pandemic. https://www.cdc.gov/coronavirus/2019ncov/hcp/dental-settings.html. Accessed November 16, 2020.

21. de Moraes Melo Neto CL, Bannwart LC, de Melo Moreno AL, Goiato MC. SARS-CoV-2 and dentistry - review. Eur J Dent. 2020;14(S 01):S130-S139. doi:10.1055/s-0040-1716438

22. van Doremalen N, Bushmaker T, Morris DH, et al. Aerosol and surface stability of SARS-CoV-2 as compared with SARS-CoV-1. N Engl J Med. 2020;382(16):1564-1567. doi:10.1056/NEJMc2004973

23. American Dental Association. CDC guidance for dental settings echoes ADA guidance. https://www.ada.org/en/press-room/ news-releases/2020-archives/may/cdc-guidance-for-dentalsettings-echoes-ada-guidance?utm_source $=$ cpsorg\&utm medium $=$ cpsalertbar\&utm_content $=$ ada-cdcstatement\&utm_ campaign=covid19. Accessed November 16, 2020.

24. Kampf G, Todt D, Pfaender S, Steinmann E. Persistence of coronaviruses on inanimate surfaces and their inactivation with biocidal agents. J Hosp Infect. 2020;104(3):246-251. doi:10.1016/j.jhin.2020.01.022

25. Matys J, Grzech-Leśniak K, Dominiak M. Disinfectants and devices for surface and air disinfection in dental offices. J Stoma. 2020;73(4):200-205. doi:10.5114/jos.2020.98267

26. Peditto $M$, Scapellato $S$, Marcianò A, Costa P, Oteri G. Dentistry during the COVID-19 epidemic: An Italian workflow for the management of dental practice. Int J Environ Res Public Health. 2020;17(9):3325. doi:10.3390/ijerph17093325

27. Dominiak M, Różyło-Kalinowska I, Gedrange T, et al. COVID-19 and professional dental practice. The Polish Dental Association Working Group recommendations for procedures in dental office during an increased epidemiological risk. J Stoma. 2020;73(1):1-10. doi:10.5114/jos.2020.94168

28. World Health Organization. The use of non-steroidal anti-inflammatory drugs (NSAIDs) in patients with COVID-19. https://www. who.int/news-room/commentaries/detail/the-use-of-non-steroidal-anti-inflammatory-drugs-(nsaids)-in-patients-with-COVID-19. Accessed November 17, 2020.

29. World Federation of Hemophilia. COVID-19 (coronavirus disease 2019) pandemic caused by SARS-CoV-2: Practical recommendations for people with haemophilia. https://news.wfh. org/COVID-19-coronavirus-disease-2019-pandemic-caused-bySARS-CoV-2-practical-recommendations-for-hemophilia-patients/. Accessed on July 11, 2020.

30. American Dental Association. What constitutes a dental emergency? https://success.ada.org/ /media/CPS/Files/Open\%20Files/ADA COVID19_Dental_Emergency_DDS.pdf. Accessed July 20, 2020.

31. Ather A, Patel B, Ruparel NB, Diogenes A, Hargreaves KM. Coronavirus disease 19 (COVID-19): Implications for clinical dental care. J Endod. 2020;46(5):584-595. doi:10.1016/j.joen.2020.03.008

32. Moffat RC, Yentes CT, Crookston BT, West JH. Patient perceptions about professional dental services during the COVID-19 pandemic. $J D R$ Clin Trans Res. 2021;6(1):15-23. doi:10.1177/2380084420969116

33. Jurič IB, Anić $I$. The use of lasers in disinfection and cleanliness of root canals: A review. Acta Stomatol Croat. 2014; 48(1):6-15. doi:10.15644/asc48/1/1

34. Suh Y, Patel S, Kaitlyn R, et al. Clinical utility of ozone therapy in dental and oral medicine. Med Gas Res. 2019;9(3):163-167. doi:10.4103/2045-9912.266997 
35. Guo YR, Cao QD, Hong ZS, et al. The origin, transmission and clinical therapies on coronavirus disease 2019 (COVID-19) outbreak - an update on the status. Mil Med Res. 2020;7(1):11. doi:10.1186/s40779-020-00240-0

36. Sabino-Silva R, Gomes Jardim AC, Siqueira WL. Coronavirus COVID-19 impacts to dentistry and potential salivary diagnosis. Clin Oral Investig. 2020;24(4):1619-1621. doi:10.1007/s00784-020-03248-x

37. Wang L, Wang Y, Ye D, Liu Q. Review of the 2019 novel coronavirus (SARS-CoV-2) based on current evidence. Int J Antimicrob Agents. 2020;55(6):105948. doi:10.1016/j.ijantimicag.2020.105948

38. Royal College of Surgeons of England. Recommendations for Dental Diagnostic Imaging during the recovery phase of the COVID-19 pandemic. https://www.rcseng.ac.uk/-/media/files/rcs/fds/guidelines/v2_recommendations-for-dental-radiography_june2020.pdf. Accessed November 21, 2020.

39. Martínez-Rider R, Garrocho-Rangel A, Márquez-Preciado R, BolañosCarmona MV, Islas-Ruiz S, Pozos-Guillén A. Dental management of a child with incidentally detected hemophilia: Report of a clinical case. Case Rep Dent. 2017;2017:7429738. doi:10.1155/2017/7429738

40. Lewandowski B, Wojnar J, Brodowski R, Mucha M, CzenczekLewandowska $E$, Brzęcka D. Dental extractions in patients with mild hemophilia $A$ and hemophilia $B$ and von Willebrand disease without clotting factor supplementation. Pol Arch Intern Med. 2018;128(7-8):488-490. doi:10.20452/pamw.4298

41. Nagarakanti S, Sappati H, Gunupati S, Ramesh Reddy BV, Chava VK Dental management of a patient with incidentally detected hemophilia: Report of a clinical case. J Indian Soc Periodontol. 2019;23(3):281-283. doi:10.4103/jisp.jisp_461_18

42. Dudeja PG, Dudeja KK, Lakhanpal M, Ali S. Endodontic management of a haemophilic patient - a clinical perspective. J Clin Diagn Res. 2014;8(7):ZD17-ZD18. doi:10.7860/JCDR/2014/8404.4592

43. Abed $H$, Ainousa A. Dental management of patients with inherited bleeding disorders: A multidisciplinary approach. Gen Dent. 2017;65(6):56-60

44. Anderson JAM, Brewer A, Creagh D, et al. Guidance on the dental management of patients with haemophilia and congenital bleeding disorders. Br Dent J. 2013;215(10):497-504. doi:10.1038/sj.bdj.2013.1097

45. Dougall A, Apperley O, Smith G, Madden L, Parkinson L, Daly B. Safety of buccal infiltration local anaesthesia for dental procedures. Haemophilia. 2019;25(2):270-275. doi:10.1111/hae.13695

46. Matys J, Grzech-Leśniak K. Dental aerosol as a hazard risk for dental workers. Materials (Basel). 2020;13(22):5109. doi:10.3390/ma13225109

47. Al-Halabi M, Salami A, Alnuaimi E, Kowash M, Hussein I. Assessment of paediatric dental guidelines and caries management alternatives in the post COVID-19 period. A critical review and clinical recommendations. Eur Arch Paediatr Dent. 2020;21(5):543-556. doi:10.1007/s40368-020-00547-5

48. Li RWK, Leung KWC, Sun FCS, Samaranayake PL. Severe acute respiratory syndrome (SARS) and the GDP. Part II: Implications for GDPs. Br Dent J. 2004;197(3):130-134. doi:10.1038/sj.bdj.4811522w 